\title{
Determination of the complete genomic sequence of Neofusicoccum luteum mitovirus 1 (NLMV1), a novel mitovirus associated with a phytopathogenic Botryosphaeriaceae
}

\author{
Armelle Marais $^{1} \cdot$ Aurélia Nivault $^{2} \cdot$ Chantal Faure $^{1} \cdot$ Sébastien Theil $^{1}$. \\ Gwenaëlle Comont $^{2} \cdot$ Thierry Candresse $^{1} \cdot$ Marie-France Corio-Costet $^{2}$
}

Received: 8 January 2017/Accepted: 13 February 2017/Published online: 27 April 2017

(c) The Author(s) 2017. This article is an open access publication

\begin{abstract}
Neofusicoccum luteum species belongs to the Botryosphaeriaceae family and is involved in grapevine wood decay diseases. The present study reports the discovery and the molecular characterization of a novel mitovirus infecting this fungus. Double-stranded RNAs were purified from cultivated $N$. luteum and analysed by next generation sequencing. Using contigs showing BlastX homology with the RNA-dependent RNA polymerase (RdRp) gene of various members of the family Narnaviridae, a single contig of approximately $1.2 \mathrm{~kb}$ was constructed. The genomic sequence was completed and phylogenetic analyses indicated that this virus represents a new member of the genus Mitovirus, for which the name of "Neofusicoccum luteum mitovirus 1 " is proposed. The genome is 2,389 nucleotides long and, based on the fungal mitochondrial genetic code, it encodes a putative protein of 710 amino acids, homologous to the RdRps of members of
\end{abstract}

The nucleotide sequence reported in this work has been deposited in the GenBank database under the accession number KY230654.

Electronic supplementary material The online version of this article (doi:10.1007/s00705-017-3338-9) contains supplementary material, which is available to authorized users.

Armelle Marais

armelle.marais-colombel@inra.fr

1 UMR 1332 Biologie du Fruit et Pathologie, INRA, Univ. Bordeaux, CS 20032, 33882 Villenave d'Ornon Cedex, France

2 UMR 1035 Santé et Agroécologie du Vignoble, INRA, Bordeaux Sciences Agro, CS 20032, 33882 Villenave d'Ornon Cedex, France the Narnaviridae family. The neofusicoccum luteus mitovirus 1 (NLMV1) RdRp contains the six conserved motifs previously reported for mitoviral RdRps. Our findings represent the first evidence that a mycovirus can infect $N$. luteum, an important pathogenic fungus of grapevine.

\section{Introduction}

Since the first discovery of viruses in Agaricus bisporus [1], mycoviruses have been described in all major taxa of fungi. Most of them have either double-stranded or single-stranded RNA genomes, and a few have a DNA genome [2]. With the expansion of novel sequencing technologies, the number of fungal viruses identified has dramatically increased in the last couple of years. Mitoviruses seem to be widespread among phytopathogenic fungi and many putative new viral species of the genus Mitovirus have been proposed [3-6]. Taxonomically, mitoviruses are members of the family Narnaviridae, which comprises the genera Mitovirus and Narnavirus. They are considered as the simplest mycoviruses, in that they contain a single linear molecule of positive-sense RNA, with a single open reading frame (ORF) encoding the viral RNA-dependent RNA polymerase $(\mathrm{RdRp})$ in the mitochondrial genetic code. Mitoviruses do not form particles, but form lipid membrane-bound vesicles, and are confined to the mitochondria of their host cells, in contrast to narnaviruses, which replicate in the cytosol [7]. Several mycoviruses, including some mitoviruses $[7,8]$ have been described to confer hypovirulence to their phytopathogenic fungal host, providing a way to develop biocontrol strategies [2]. 


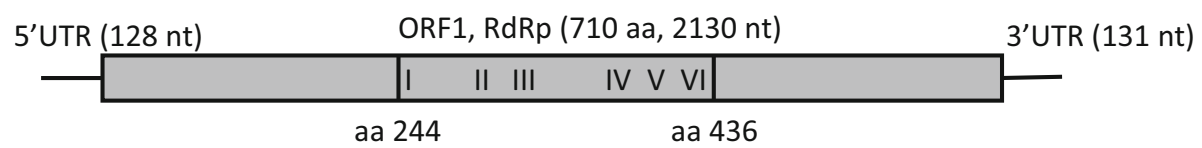

Fig. 1 Schematic representation of the genome organization of neofusicoccum luteum mitovirus 1 . The open reading frame 1 (ORF1) encoding the RNA-dependent RNA polymerase is shown as the rectangular box, as well as the conserved region with the six

Neofusicoccum luteum is involved in Botryosphaeria dieback, a worldwide grapevine trunk disease [9]. In the present study, we report the discovery of a novel species of mitovirus infecting $N$. luteum for which the name of "Neofusicoccum luteum mitovirus 1 " is proposed. The complete genomic sequence of neofusicoccum luteum mitovirus 1 (NLMV1) was determined and consists of a 2,389 nucleotides long RNA molecule encoding a putative RdRp of 710 amino acids (aa).

\section{Virus material}

The $N$. luteum isolate used was NL-37 (GWEMA-UMR SAVE collection), sampled in a vineyard in 1996, on canes of Vitis vinifera. The fungus was grown as described previously [10].

\section{Results}

Double-stranded RNAs were purified and analyzed on agarose prior to random amplification and Miseq sequencing in a multiplexed scheme as previously reported [11]. After quality trimming and demultiplexing, a total of 136,741 paired reads were obtained, assembled using home-made pipelines, and compared to GenBank database using BlastN and BlastX. A contig of approximately 1.2 $\mathrm{kb}$, integrating $2.3 \%$ of the total reads, revealed significant similarities with various members of the genus Mitovirus. The 5' and 3' genome ends were determined using purified dsRNA as template and 5' and 3' Rapid Amplification of cDNA Ends (RACE) with internal primers designed from the contig, following the kit manufacturer's instructions conserved motifs typically observed in mitoviral RdRps (I, II, III, IV, $\mathrm{V}$, and VI). The amino acid position of the start and end of the conserved region is also shown. UTR, Untranslated region

(Takara Bio Europe/Clontech, Saint-Germain-en-Laye, France).

The complete NLMV1 genome sequence is 2,389 nt long (GenBank accession number KY230654), which is a typical size for a mitoviral genome [12]. Its GC content is relatively low (30.6\%), which is also characteristic of members of the genus Mitovirus. The genetic organization of the genome is shown in Fig. 1. It contains a 128 nt long 5'-untranslated region (UTR) and a 3'-UTR of $131 \mathrm{nt}$, and, as most mitoviruses, has no 3' poly(A) tail. As expected for a mitovirus, the 5'- and 3'UTRs of NLMV1 could be folded into two stable stemloop structures with $\Delta G$ values of $-35.7 \mathrm{kcal} / \mathrm{mol}$ and $-52.3 \mathrm{kcal} / \mathrm{mol}$, respectively. In contrast, no inverted complementarity between the two UTRs was detected, suggesting absence of a panhandle structure. This has been described in other mitoviruses, such as alternaria arborescens mitovirus 1 [6]. Using the mitochondrial genetic code, a single large ORF was detected, encoding a 710 aa long polypeptide, sharing significant but weak sequence similarity with the RdRp gene of several mitoviruses. The most closely related species was Sclerotinia sclerotiorum mitovirus 18 , with $38.4 \%$ of aa sequence identity. Considering the species demarcation criteria accepted for the genus Mitovirus (less than $40 \%$ of aa identity in the RdRp gene, [12]), NLMV1 should be considered as a member of a new species within this genus. A neighbor-joining tree was reconstructed with the RdRp sequences of members of the family Narnaviridae (Fig. 2) and showed that NLMV1 clusters with the Clade II of mitoviruses. As expected, analysis of the conserved domains in the NLMV1 RdRp revealed the presence of the six motifs (I to VI) which are typical of mitoviral RdRps [13]. 
Fig. 2 Neighbor-joining phylogenetic tree reconstructed using the complete amino acid sequences of RNA-dependent RNA polymerases from members of the family Narnaviridae. The tree uses strict identity distance and the statistical significance of branches was evaluated by bootstrap analysis $(1,000$ replicates). Only values higher than $70 \%$ are indicated. The scale bar represents $10 \%$ amino acid divergence. The sequence of the neofusicoccum luteum mitovirus 1 is marked by a black pentagon

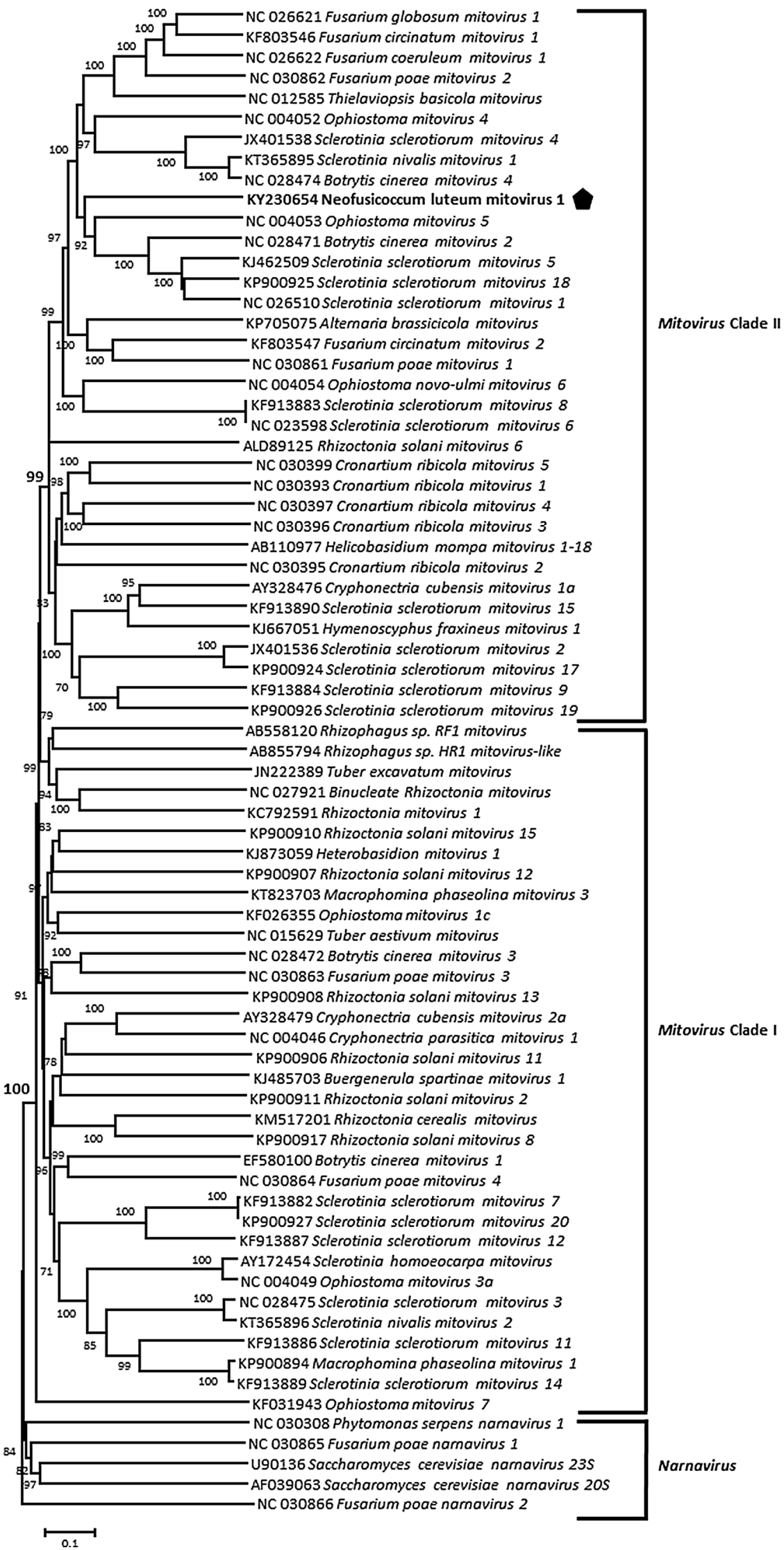


Some mitoviruses have been shown to affect the virulence of their hosts [8]. This study is the first report of a mitovirus infecting $N$. luteum involved in grapevine Botryosphaeria dieback. Further studies are needed to assess the virulence of NLMV1, and to explore the overall diversity of viruses infecting this pathogenic fungus.

Acknowledgements The authors thank the Platform Genotoul (INRA, Toulouse, France) for the Illumina sequencing.

\section{Compliance with ethical standards}

Conflict of interest All authors declare no conflict of interest.

Ethical approval This article does not contain any studies with animals or human participants performed by any of the authors.

Open Access This article is distributed under the terms of the Creative Commons Attribution 4.0 International License (http://crea tivecommons.org/licenses/by/4.0/), which permits unrestricted use, distribution, and reproduction in any medium, provided you give appropriate credit to the original author(s) and the source, provide a link to the Creative Commons license, and indicate if changes were made.

\section{References}

1. Hollings M (1962) Viruses associated with a die-back disease of cultivated mushroom. Nature 196:962-965

2. Ghabrial SA, Caston JR, Jiang D, Nibert ML, Suzuki N (2015) 50-plus years of fungal viruses. Virology 479-480:356-368

3. Osaki H, Sasaki A, Nomiyama K, Tomioka K (2016) Multiple virus infection in a single strain of Fusarium poae shown by deep sequencing. Virus Genes 52:835-847
4. Marzano SL, Nelson BD, Ajayi-Oyetunde O, Bradley CA, Hughes TJ, Hartman GL, Eastburn DM, Domier LL (2016) Identification of diverse mycoviruses through metatranscriptomics characterization of the viromes of five major fungal plant pathogens. J Virol 90:6846-6863

5. Liu JJ, Chan D, Wiang Y, Williams H, Li XR, Sniezko RA, Sturrock RN (2016) Characterization of five novel mitoviruses in the white pine blister rust fungus Cronartium ribicola. PLoS One 11:e0154267. doi:10.1371/journal.pone.0154267

6. Komatsu K, Katayama Y, Omatsu T, Mizutani T, Fukuhara T, Kodama M, Arie T, Teraoka T, Moriyama H (2016) Genome sequence of a novel mitovirus identified in the phytopathogenic fungus Alternaria arborescens. Arch Virol 161:2627-2631

7. Hillman BI, Cai G (2013) The family Narnaviridae: Simplest of RNA viruses. Adv Virus Res 86:149-176

8. Xu A, Wu S, Liu L, Cheng J, Fu Y, Jiang D, Xie J (2015) A mitovirus related to plant mitochondrial gene confers hypovirulence on the phytopathogenic fungus Sclerotinia sclerotiorum. Virus Res 197:127-136

9. Phillips AJL (2002) Botryosphaeria species associated with diseases of grapevines in Portugal. Phytopathologia Mediterranea 41:3-18

10. Bellée A, Comont G, Nivault A, Abou-Mansour E, Coppin C, Dufour MC, Corio-Costet MF (2016) Life traits of four Botryosphaeriacea species and molecular responses of different genotypes and cultivar of Vitis vinifera. Plant Pathol. doi:10. 1111/ppa.12623

11. Candresse T, Marais A, Faure C, Gentit P (2013) Association of Little cherry virus 1 with the Shirofugen stunt disease and genome characterization of a divergent LChV1 isolate. Phytopathology 103:293-298

12. Hillman BI, Esteban R (2012) Family Narnaviridae. In: King AMQ, Adams MJ, Carstens EB, Lefkowitz EJ (eds) Virus taxonomy-ninth report on the International Committee on Taxonomy of Viruses. Elsevier, San Diego, pp 1055-1060

13. Hong Y, Dover SL, Cole TE, Brasier CM, Buck KW (1999) Multiple mitochondrial viruses in an isolate of the Dutch elm disease fungus Ophiostoma novo-ulmi. Virology 258:118-127 